\title{
Die näherungsweise Lösung der elektrostatischen Aufgabe mit der Finite - Element - Methode zur Modellierung behinderter elektrischer Entladungen
}

\author{
Andreas Harendt, Harald. J. Beyer, Siegfried Rolle
}

\section{Zusammenfassung}

Es wird erläutert, warum man mit der Finite-ElementMethode (FEM) das elektrostatische Problem mit Grenzflächen und darauf befindlichen Flächenladungen prinzipiell nicht lösen kann. Es werden dann Verfahren entwickelt, dies näherungsweise zu tun. Anschließend wird eine Methode entwickelt, die es gestattet, aus den so erhaltenen Lösungen näherungsweise konsistent auf die Ausgangsgleichungen zurïckzurechnen und so den Driftterm der Kontinuitätsgleichung für die hydrodynamische Modellierung einer Gasentladung zu ermitteln.

\section{Problemstellung und Motivation}

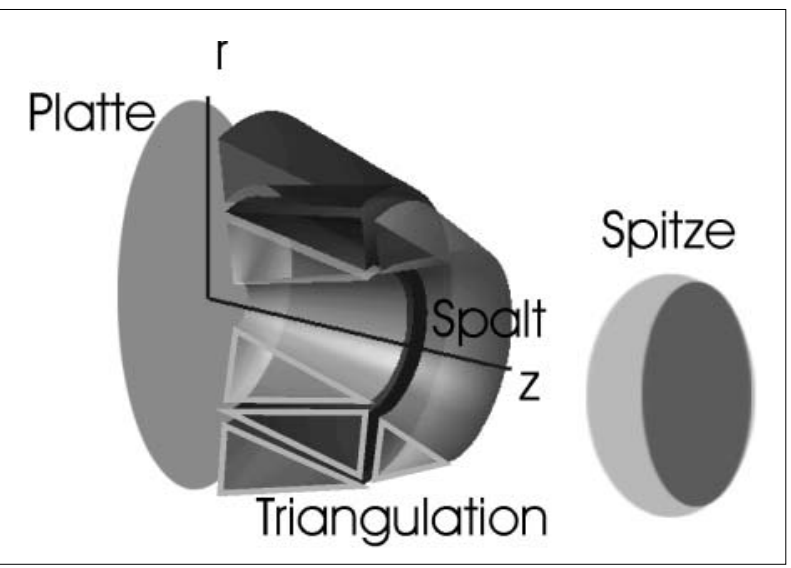

Abb. 1: Zur Triangulation in Zylindersymmetrie

Im Rahmen eines Verbundprojektes bestand u. a. die Aufgabe, eine behinderte elektrische Entladung (den Funken) zwischen einer dielektrischen Platte und einer ebenso dielektrischen Spitze zu modellieren. Der Abstand zwischen der Spitze und der Platte liegt dabei zwischen $10-100 \mu \mathrm{m}$. Die relativen Dielektrizitätskonstanten der beiden Elektroden in der Größenordnung $10^{3}$. Modellierungen solcher Entladungen wurden bereits vorher unternommen, aber immer in finiten Differenzen. Da die Krümmung in der Geometrie berüicksichtigt werden sollte, wurde auf die FEM orientiert, da diese eine leichte Anpassbarkeit an gegebene Geometrien gestattet.

Aus der Abb. 1 ist auf sehr anschauliche Weise die Geometrie und deren Symmetrie zu ersehen: Senkrecht zur mit $z$ bezeichneten Achse befinden sich die beiden rotationssymmetrischen Dielektrika (Platte und Spitze). Die $z$-Achse durchstößt diese als Rotationsachse. Um diese Rotationsachse legt man nun im Spalt, dem Entladungsraum, Rotationskörper von dreieckigem Querschnitt, die so aneinandergelegt sind, dass sie den Raum zwischen Platte und Spitze vollständig ausfuillen.

Nun setzt man im Folgenden die zu erwartenden Lösungen als unabhängig vom Winkel voraus. Dann kann das eigentlich dreidimensionale Problem auf ein zweidimensionales reduziert werden, indem man etwa in der $r$-z-Ebene einen Schnitt tätigt. Übrig bleibt fuir die Diskretisierung ein triangulares Netz.

\section{Physikalische Grundlagen}

Im Spalt ist dann zur Berechnung der Ladungsträgerdichten die Kontinuitätsgleichung

$$
\frac{\partial \mathrm{N}_{k}}{\partial t}=-\operatorname{div}\left(v_{D}^{k} \mathrm{~N}_{k}\right)+\mathrm{Q}_{k}
$$

für jede Spezies $k$ zu lösen. $N_{k}$ ist die Dichte der jeweiligen Teilchensorte, $\vec{v}_{D}^{k}$ die am Ort herrschende Driftgeschwindigkeit für diese und $Q$ ein Produktions- bzw. Destruktionsterm. Die beiden letzteren Größen kann man bei den hier herrschenden Neutralgasdichten als explizite Funktionen des Betrages der elektrischen Feldstärke annehmen. $Q$ ist dabei aber auch noch eine Funktion der Teilchendichten $N_{k}$. Im einfachsten Fall wäre

Das Vorzeichen entspricht der Ladungsträgerpolarität und $b$ heißt die Beweglichkeit. Diese kann bei gegebener Neutralgasdichte in erster Näherung als konstant angenommen werden. Mit dem Ausdruck

$$
\frac{\alpha}{N_{0}}=A e^{-\frac{B}{\left|E / N_{0}\right|}}
$$

für den Townsendschen Ionisierungskoeffizienten $\alpha$, der die Zahl der durch inelastische Elektronenstöße neu erzeugten Ladungsträgerpaare, bezogen auf die zurückgelegte Wegstrecke angibt ( $A$ und $B$ sind von der Gasart abhängige Konstanten und $N_{0}$ die Neutralgasdichte), kann man für den Produktionsterm der Elektronen und Ionen

$$
Q_{e, i}=\alpha\left|v_{D}^{e, i}\right| N_{0} N_{e}-R N_{i} N_{e}
$$

schreiben. Darin ist noch $R$ eine Rekombinationsrate, die hier als konstant angenommen werden kann. Mit den Be- 
ziehungen (2) und (4) lässt sich (1) lösen, wenn man die elektrische Feldstärke kennt und man in der Lage ist, daraus

$$
\operatorname{div}\left(\vec{v}_{D}^{k} N_{k}\right)
$$

konsistent (numerisch!) zu berechnen. Wenn dies bei den zu beobachtenden (und auch berechneten) Stromanstiegsgeschwindigkeiten und den auch beträchtlichen Stromdichten möglicherweise bereits recht grob ist, so muss hier auf das System der quasistationären Maxwellschen Gleichungen zurüickgegriffen werden. Dieses folgt aus der Annahme, dass $\partial \vec{B} / \partial t$, die zeitliche Änderung der magnetischen Induktion hinreichend klein ist. In diesem Fall kann man

setzen. Gilt nun (6), dann lässt sich bekanntlich die elektrische Feldstärke $\vec{E}$ als Gradient eines skalaren Potentials $U$ darstellen:

Mit der Gleichung

für die durch die Raumladungen $\rho$ erzeugte dielektrische Erregung $\vec{D}$ und der Materialgleichung

erhält man dann

$$
-\operatorname{div}(\varepsilon \operatorname{grad} U)=\rho,
$$

die im Falle konstanten $\varepsilon$ in die Poissongleichung übergeht. Aus (8) folgt aber auch, dass an den Grenzfächen zweier Medien mit unterschiedlichem $\varepsilon$, etwa $\varepsilon_{1}$ und $\varepsilon_{2}$, die Bedingung

$$
\left(\vec{D}_{1}-\vec{D}_{2}\right) \cdot \vec{n}=\eta
$$

gelten muss. Dabei sind unter $\vec{D}_{1,2}$ die Grenzwerte der Erregung bei Annäherung aus dem jeweiligen Gebiet an die Grenzfläche zu verstehen, ist hier der Normalenvektor der Grenzfläche, in das Gebiet 1 weisend und $\eta$ eine sich auf der Grenzfläche befinden könnende Flächenladung. Nun gehen die Größen aus (1) selbst wieder in die Gleichungen der Elektrodynamik ein. Das $\rho$ in (10) ist

Hinzu kommt noch, dass Flüsse aus (1) auch noch Änderungen der rechten Seite von (11) bewirken. Damit ist das System selbstkonsistent formulierbar.' So man nun (z. B. für stetig veränderliches $\varepsilon$ ) Gl. (10) lösen kann, heißt dies nicht, dass man damit das elektrostatische Problem mit unstetigen Änderungen der Dielektrizitätskonstanten und Flächenladungsbelegungen innerhalb von der FEM bewältigen kann.

\section{Korrekfur der FEM an Grenzflächen}

Mit einem Netz hoher Qualität (s. u.) ist Gl. (10) für das von uns zu behandelnde Problem gelöst worden, und das Ergebnis in Abb. 2 gezeigt.

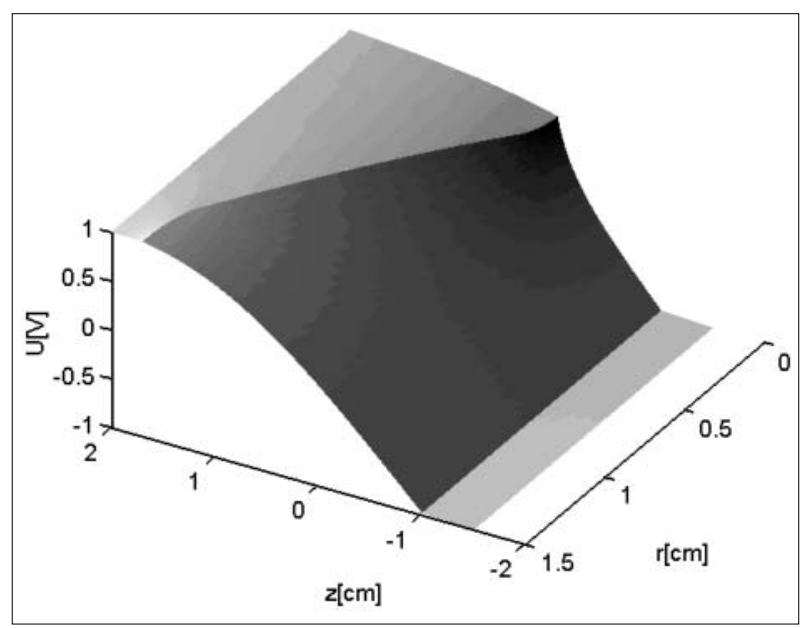

Abb. 2: Beispiel für Potential und Betrag der elektrischen Feldstärke: Die Höhe entspricht dem Potential, die Schwärzung dem $\log (|\mathrm{E}|)$.

In der Platte (hier rechts) und der Spitze (hier links) findet man aufgrund der hohen relativen Dielektrizitätskonstanten $\left(\varepsilon_{L}=\varepsilon_{R}=20\right.$ und im Spalt $\left.\varepsilon_{S}=1\right)$ nur ein schwaches elektrisches Feld. An der Spitze zum Spalt hin natürlich erwartungsgemäß ein Maximum des Betrages desselben.

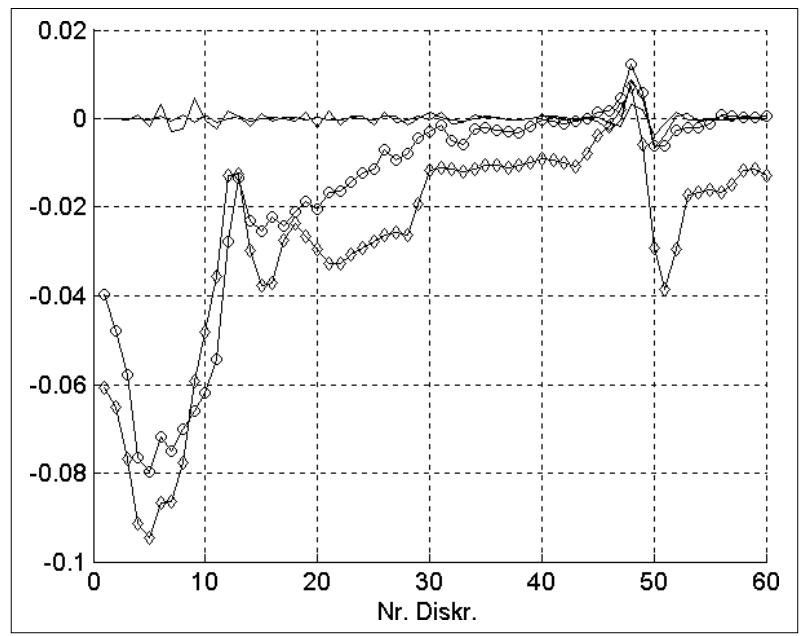

Abb. 3: Fehler bezüglich (11) für zwei verschiedene Interpolationsverfahren ohne (Kurve mit Markern) und mit Korrektur.

Die Abb. 3 zeigt den Fehler, den die FEM bei der Lösung von Gl. (10) bezüglich der Erfüllung von Gl. (11) mit $\eta=0$ verursacht. Gezeigt wird die Differenz von $\varepsilon_{S} \vec{E} \cdot \vec{n} / \max |E|$ und $\varepsilon_{R} \vec{E} \cdot \vec{n} / \max |E|$ (wobei die relativen Dielektrizitätskonstanten genutzt wurden). An kaum einer Stelle der Grenzfläche ist die elektrostatische Aufgabe auch nur hinreichend bezuiglich der Nebenbedingung (11) erfuillt. Eine Verfeinerung des Netzes belässt den dabei erzeugten Fehler in der Größenordnung bis zu 10 \%." Dabei sind die Normalkomponenten mit Hilfe zweier Interpolationsmechanismen gebildet worden. Diese nutzen auf unterschiedliche Weise mehrere Feldstärkewerte aus der Umgebung eines Grenzflächen- 
stuickes und ermitteln daraus die jeweiligen Normalkomponenten in Extrapolation auf die Grenzflächen. Darin liegt natürlich eine gewisse Willkür. Wählt man zur Beurteilung einen anderen Interpolations-Extrapolationsmechanismus, dann erhält man auch andere Fehler.

Ursprüngliches Ziel war eine Möglichkeit zu finden, Flächenladungen in der FEM zu berïcksichtigen. Der Gedanke war nun folgender: Wenn die FEM einen Fehler bzgl. (11) verursacht, dann muss sich dieser Fehler korrigieren lassen, indem man in einer kleinen Umgebung der Grenzfläche eine geeignet gewählte Raumladung für die rechte Seite von (10) so wählt, dass man (11)

1. für $\eta=0$ besser befriedigen kann und

2. für $\eta \neq 0$ dann natürlich auch Lösungen findet.

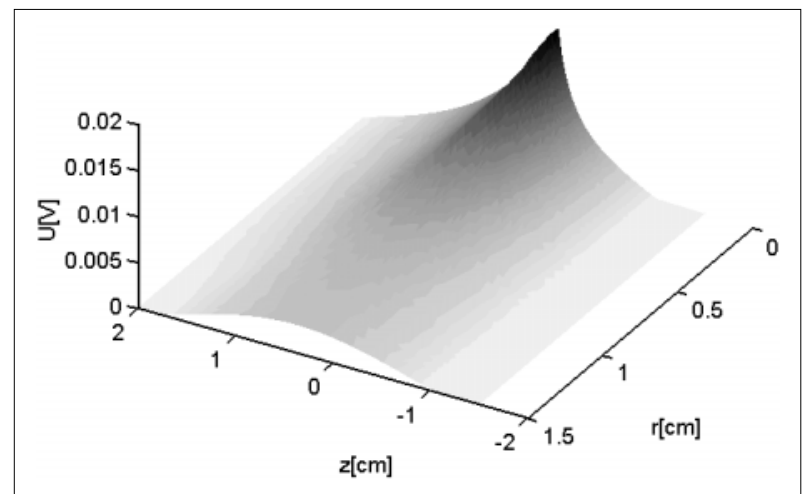

Abb. 4: Korrekturpotential

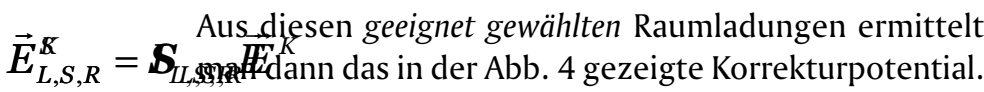
Vergleicht man mit den Potentialwerten aus der Abb. 2, dann ist ersichtlich, dass es sich wirklich nur um eine kleine Korrektur handelt. Addiert man diese allerdings zum Potential und untersucht dann die Gültigkeit von (11), so erhält man das in Abb. 3 gezeigte Ergebnis der Korrektur. Der Fehler konnte um gut eine Größenordnung reduziert werden und verringert sich mit feiner werdender Diskretisierung weiter. Wie erhält man nun geeignet gewählte Raumladungen bzw. das Korrekturpotential?

Die FEM ermittelt aus der Beschreibung der Geometrie, den Randbedingungen, dem Triangulationsnetz und der Angabe von $\varepsilon$ für den Mittelpunkt eines jeden Dreiecks die Matrizen $K, F$ und $B$, mit deren Hilfe sich für beliebige rechte Seiten in (10) und den Dirichletschen Randwerten $U_{d}$ das Potential $U$ nach

$$
U_{n d}=\boldsymbol{K}^{-1}\left(\boldsymbol{F} \rho+\boldsymbol{B} U_{d}\right)
$$

fuir die nicht Dirichletschen Knotenpunkte $U_{n d}$ der Triangulation (näherungsweise) berechnen lässt.

Die elektrische Feldstärke erhält man als Gradienten jedes der Triangulationsdreiecke (siehe auch Abb. 11 oder die hellgrauen Pfeile in Abb. 10) aus

$$
\vec{E}=\boldsymbol{G}_{r} U \vec{e}_{r}+\boldsymbol{G}_{z} U \vec{e}_{z}
$$

mit den Interpolationsmatrizen $G_{r}$ und $G_{z}$. Grundsätzlich gilt der nach Gl. (14) berechnete Wert des Gradienten auf der gesamten Fläche eines einzelnen Triangulations- elements. Es liegt nun wieder eine gewisse Willkür darin, wie man aus diesen Werten zurïck auf die Knotenpunkte interpoliert. Nimmt man aber an, dass die Wahrscheinlichkeit für die Richtigkeit des aus der linearen Formel (14) ermittelten Gradienten im Schwerpunkt des Triangulationsdreiecks am höchsten ist, liegt Folgendes nahe: Die Abstände eines Knotenpunktes zu den Schwerpunkten der ihn umgebenden Triangulationselemente seien $l_{i}$, mit $i=1 \ldots n$. Dann kann man als Interpolationsformel das gewichtete Mittel für die Knotenpunkte (die dunkelgrauen Pfeile in Abb. 10) aus

$$
\vec{E}_{j}^{K}=\sum_{i=1}^{n} \frac{1}{l_{i}} \vec{E}_{i}^{S} / \sum_{i=1}^{n} \frac{1}{l_{i}}=\sum_{i=1}^{n} w_{i} \vec{E}_{i}^{S}
$$

ermitteln, oder in Matrixschreibweise mit den drei Interpolationsmatrizen $I_{L, S, R}$ als

Alle Knotenpunkte sind aber durch das Triangulationsnetz miteinander verbunden, und damit kann man für jede Koordinate innerhalb des Netzes die Werte von $\vec{E}$ durch lineare Interpolation ermitteln, kann also letztendlich in einer beliebig gewählten Umgebung der Grenzfläche ein Stuitzstellensystem

zur Interpolation zwecks Extrapolation der Normalkomponenten der dielektrischen Erregung bilden, und erhält an den Grenzflächen die der Gl. (11) entsprechenden Bedingungen

$$
\boldsymbol{A}_{S} D_{n S}^{S}-\boldsymbol{A}_{L, R} D_{n L, R}^{S}=\eta
$$

für jedes Grenzflächenelement. Darin sind $A_{S, L, R}$ wieder Matrizen, die in diesem Fall den Extrapolationsprozess beschreiben.

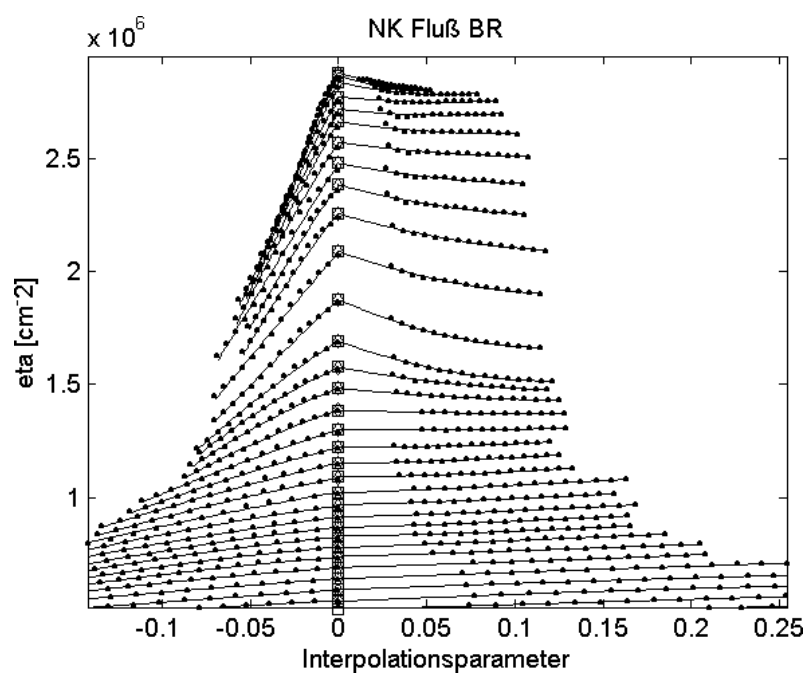

Abb. 5: Interpolation und Extrapolation der Normalkomponenten des dielektrischen Flussen $\mathrm{D}_{\mathrm{n}}$ auf die Grenzfläche bei korrigierter Lösung. ${ }^{\text {II }}$

In der Abb. 5 ist ein solcher Interpolations-Extrapolationsmechanismus gezeigt. Zu jedem Grenzflächenelement gehört ein links- und rechtseitiger Satz von 
Stuitzstellen. Jeder dieser Stuitzstellen ist ein Wert des Interpolationsparameters $\xi$ zugeordnet. Jeder Punkt entspricht dabei der Normalkomponenten in dem Stuitzstellensystem. Für den Spalt wurde über $-\xi$ aufgetragen. Innerhalb der Barriere über $+\xi$, so dass bei $\xi=0$ das Zusammentreffen der Grenzwerte stattfindet. Durch diese Stuitzstellen sind die Interpolationskurven gelegt und die Extrapolation auf $\xi=0$ ist ebenso gezeigt.

Im unkorrigierten Fall hat man, wie in der Abb. 3 dargestellt, kein Zusammentreffen der Kurven bei $\xi=0$. Man muss also an Stelle von Gl. (18)

$$
\boldsymbol{A}_{S} D_{n S}^{S}-\boldsymbol{A}_{L, R} D_{n L, R}^{S}=\eta+\delta
$$

schreiben, worin d der Fehler ist. Nun wurde schon vorgefuihrt, dass man durch eine geeignete Wahl von Korrekturraumladungen $\rho_{k}$ diesen Fehler $\delta$ verringern kann. Da alle Gl. in der Folge (13) - (19) linear sind, ergeben sich die $\rho_{k}$ aus

$$
-\boldsymbol{J} \rho_{k}=\left.\delta\right|_{\rho_{k}=0}+\eta \cdot
$$

Darin ist $J$ die Jacobimatrix der linken Seite von Gl. (19). Bei $m$ Grenzflächen, den dazu gehörenden Fehlern $\delta_{1 \ldots m}\left(\rho_{1 \ldots n}\right)$ und bei $n$ zu ermittelnde Korrekturladungen $\rho_{1 \ldots n}$ hat sie die Form

Sie muss (wegen der durchgehenden Linearität der numerischen Lösung) nur einmal für eine gegebene Geometrie und die dazugehörigen Materialparameter ermittelt werden. Es bleibt als letzter zu behandelnder Gegenstand in diesem Punkt die Frage: Wie viele Korrekturladungen sind anzubringen?

Offensichtlich gibt es $n$ Grenzflächen. Nun besitzt jede dieser Grenzflächen genau zwei an ihr anliegende Dreiecke des Triangulationsnetzes. Nun könnte man eines davon (z. B. im Entladungsspalt oder in der Barriere) auswählen, um die Korrekturen dort anzubringen. Man hätte dann den einfachen Fall, genau soviel Korrekturladungen anbringen zu müssen, wie Gleichungen vorhanden sind und es wäre im flächenladungsfreien Fall einfach

$$
\rho_{k}=-\left.\boldsymbol{J}^{-1} \delta\right|_{\rho_{k}=0} .
$$

Dies hätte zur Folge, das letztendlich Gl. (11) bzw. (18) exakt erfuillt werden würden. Dies hört sich verlockend an - und funktioniert auch. Nur sind die dann erhaltenen Korrekturladungen keine Korrekturen mehr, sondern von exorbitanter Größe und das erhaltene Korrekturpotential in oder über der Größenordnung des eigentlichen Potentials. Hier hat sich nun nach langem experimentieren ein Kompromiss als günstig erwiesen:
- Man verteilt die Korrekturen über mehr als $n$ Dreiecke.

- Dies fuihrt dann zu einem unterbestimmten System.

- Deshalb legt man den Korrekturen zusätzliche Bedingungen auf - z. B. kann man näherungsweise Quasineutralität und/oder Stetigkeit zwischen benachbarten Korrekturen verlangen.

- Dies fuihrt dann - so man geschickt agiert - zu einem uiberbestimmten System, dessen Forderungen man dann im Sinne der zu minimierenden Quadrate der Fehler befriedigt. Dies soll hier aber nicht im Einzelnen dargestellt werden.

Als Bestimmungsgleichung für die Korrekturladungen erhält man in diesem Fall

$$
-\boldsymbol{J}^{T} \boldsymbol{J} \rho_{k}=\boldsymbol{J}^{T}\left(\left.\delta\right|_{\rho_{k}=0}+\eta\right) .
$$

\section{Das Verschmieren von Flächenladungen als Raumladungen}

Die numerische Behandlung von Flächenladungen im Rahmen der gegebenen Lösungsmethoden für Gl. (10) ist - so die FEM ja schon in Abwesenheit derselben nicht korrekt an Grenzflächen löst, nicht gegeben. Oben wurde gezeigt, dass man diesen Fehler verringern kann. Nun soll die Möglichkeit untersucht werden, Flächenladungen überhaupt als Raumladungen verschmieren zu können. Dazu betrachtet man das in der Abb. 6 gezeigte eindimensionale elektrostatische Problem mit einer Grenzfläche zwischen zwei Dielektrika mit den relativen Dielektrizitätskonstanten $\varepsilon_{1}$ und $\varepsilon_{2}$. Diese befinden sich ggf. in einem äußeren Potential mit der Potentialdifferenz $u=\left.U\right|_{-s}-\left.U\right|_{d}$.

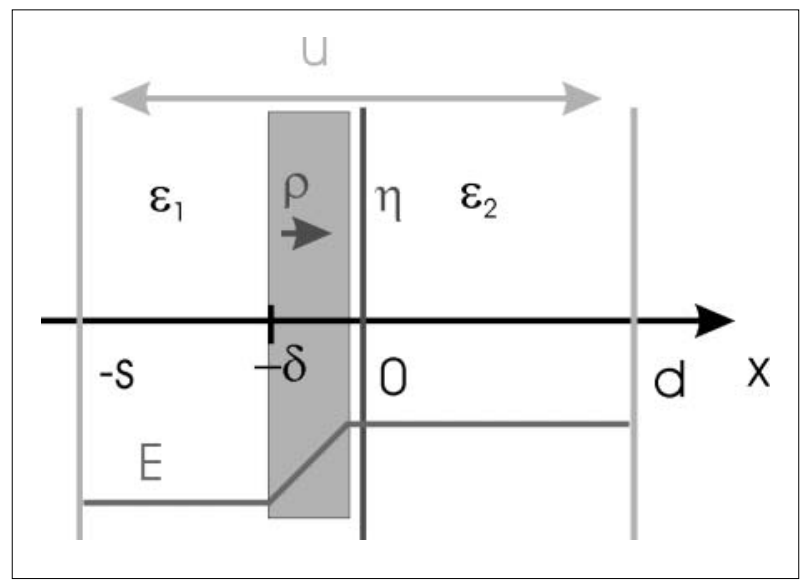

Abb. 6: Geometrie zur eindimensionalen Untersuchung bzgl. der Näherung von Flächenladungen durch Raumladungen $(u=o)$.

Raumladungen seien nur zwischen $-\delta \ldots 0$. Die Flächenladung $\eta$ sei dabei entweder als Raumladung vor der Grenzfläche in einer Schicht der Dicke $\delta$ mit $\rho=\eta / \delta$ verteilt oder aber auf der Grenzfläche als solche gegeben. Mit der Abkürzung ergeben sich die in der Tab. 1 dargestellten Ausdrücke für das Wirken der gleichen Ladungsmenge jeweils als Flächen- oder Raumladung (für Details der Rechnungen s. [2]). 


\begin{tabular}{|c|c|}
\hline Flächenladung & Raumladung \\
\hline$E_{1}^{\eta}=-\frac{\eta}{\varepsilon_{1}} \frac{1}{1+g}$ & $E_{1}^{\rho}(-\delta)=-\frac{\eta}{\varepsilon_{1}} \frac{1+\frac{\delta}{2 s} g}{1+g}$ \\
\hline$E_{2}^{\eta}=\frac{\eta}{\varepsilon_{2}} \frac{1}{1+1 / g}$ & $E_{2}^{\rho}=\frac{1}{\varepsilon_{2}} \eta \frac{2 s}{1+\frac{\delta}{2 s}}$ \\
\hline
\end{tabular}

Tab. 1: Vergleich zwischen der Wirkung einer Flächenladung und derselben Ladungsmenge verschmiert als Raumladung $(u=o)$.

Man kann also jenseits der Verschmierungszone Bedingungen erzielen, wie sie durch Flächenladungen erzeugt werden, wenn man nur die aus den Gleichungen der oben gegebenen Tab. 1 geforderten Bedingungen über eine genügend kleine Breite der Verschmierungszone einhält. Innerhalb derselben sind die Verhältnisse physikalisch nicht zu hinterfragende numerische Korrekturgrößen. Die der Flächenladung entsprechende Raumladung ist hier im Gebiet 1 mit der Dielektrizitätskonstante $\varepsilon_{1}$ verteilt. Die rechte Spalte der Tab. 1 legt nun nahe, dafür die Hälfte mit der größeren Dielektrizitätskonstante zu wählen, weil dann im Medium 1 die Kleinheit von $\delta$ leichter zu befriedigen ist. Im Medium 2 muss dann $\delta$ nur klein im Verhältnis zu den sonstigen geometrischen Abmessungen gewählt werden. Dies ist der Grund, warum es vorteilhaft ist, die Korrektur- und verschmierten Flächenladungen in den Barrieren und nicht im Entladungsspalt unterzubringen.

\section{Die Berücksichtigung von Flächen- ladungen bei FEM-Lösungen des elektrostatischen Problems}

Prinzipiell wäre man mit Gl. (23) fertig. Man könnte mit dieser einfach Korrekturen berechnen, die dann die Sprungbedingung (11) an den Grenzflächen (entsprechend dem verwendeten Interpolations-Extrapolationsverfahren) näherungsweise befriedigen. Da aber in jedem Schritt Gl. (10) bzw. ihr FEM-Pendant (13) egal zwei mal gelöst werden muss und oben festgestellt wurde, dass man eine Flächenladung verschmieren kann, berücksichtigen wir dies bereits im ersten Schritt. Im zweiten Schritt berechnen wir dann nach (23) die Korrekturladungen und für diese mit (13) das Korrekturpotential, dass dann im Prinzip wieder klein sein muss. Dies gibt eine bessere Kontrolle über die Stabilität des Verfahrens.

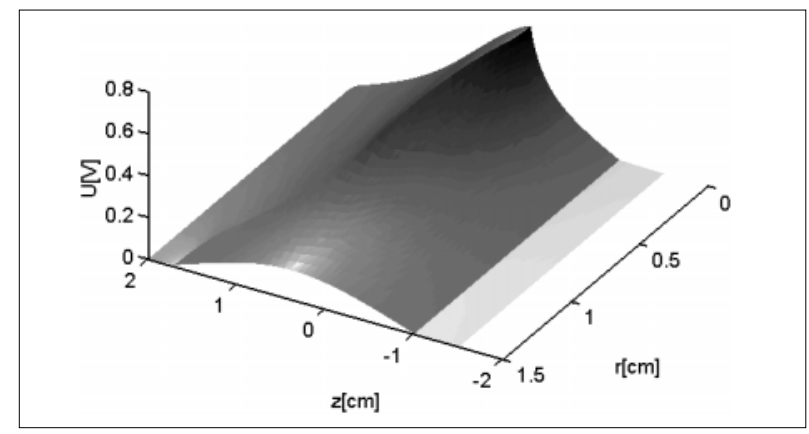

Abb. 7: Potential und $\log /$ E/ einer Flächenladung
Die Abb. 7 zeigt nun das so ermittelte Potential und den Betrag der Feldstärke als Schwärzung für eine auf einem Teil der Spitze aufgebrachten Flächenladung von $2,510^{6} \mathrm{~cm}^{-2}$ bei einer äußeren Potentialdifferenz von 0 .

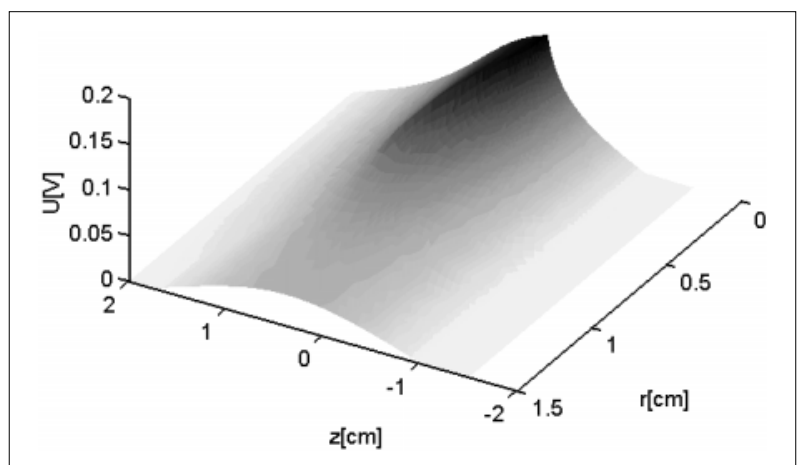

Abb. 8: Korrekturpotential zur Erfüllung von (11)

Die Abb. 8 zeigt das nach dem einfachen Verschmieren und dem oben beschriebenen Verfahren über die Ermittlung von Korrekturladungen erhaltene Korrekturpotential. Es ist klein, aber gerade an der Spitze, wo die Geometrie eine starke Krümmung hat, und die oben aus den eindimensionalen Überlegungen dargestellten Beziehungen am wenigsten gelten werden, nicht unbeträchtlich. Dazu zeigt die Abb. 9 noch die aus Gl. (11) bzw. (18) folgende Rückrechnung der Flächenladungen.

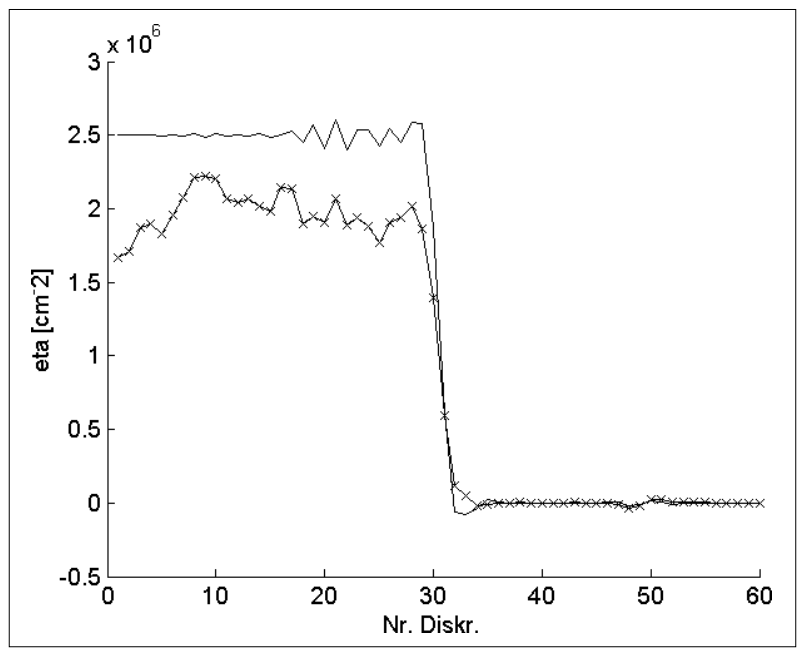

Abb. 9: Lösung der elektrostatischen Aufgabe: Die Bedingung (11) ist erfüllt.

Der markierte Linienzug zeigt diese ohne Berücksichtigung des Korrekturpotentials (also bei nur verschmierter Flächenladung). Das Ergebnis ist nicht nur stark schwankend, sondern liegt durchweg zu niedrig. Das einfach gezeichnete Resultat ist das Ergebnis bei Beruicksichtigung des Korrekturpotentials.

\section{Konstruktion hochqualitativer Triangulationsnetze}

Zur Lösung unserer eigentlichen Aufgabe, der Integration von Gl. (1) benötigt man eine numerisch konsistente Berechnung von (5). Diese ist mit (2) anzuschreiben als

$$
\operatorname{div}\left( \pm b_{k} \vec{E} N_{k}\right)
$$


Wenn $b_{k}$ als konstant angenommen wird und man $N_{k}$ mit der ortsabhängigen Dielektrizitätskonstanten assoziiert, entspricht diese Gleichung letztendlich der Quellenbedingung der Elektrostatik:

$$
\operatorname{div}(\varepsilon \vec{E})=\operatorname{div} \vec{D}=\rho
$$

Eine (numerische) Rückrechnung der mit der FEM erhaltenen Lösungen von Gl. (10) über (8) muss also wieder die in (10) stehende rechte Seite, also $\rho$ ergeben, damit dann (5) berechenbar wird. Dies erscheint auf den ersten Blick trivial - ist es aber nicht. Mit Hilfe der Abb. 10 wird dies erklärt:

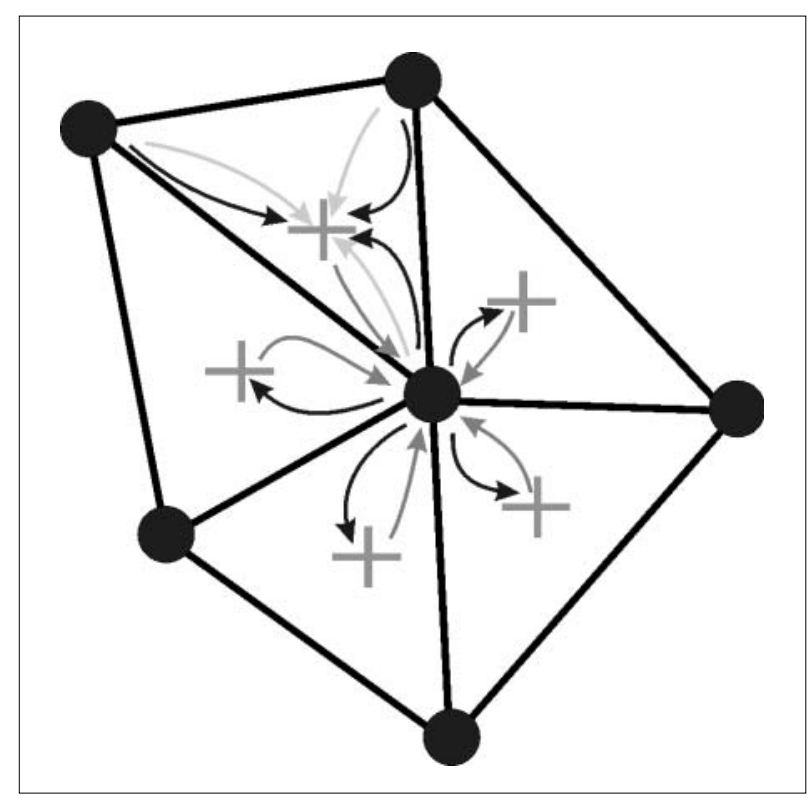

Abb. 10: Graph zum Interpolationsmechanismus der Rückrechnung aus Gl. 10

In den Knotenpunkten sind die skalaren Werte des Potentials $U$ gegeben, aus denen nun die Vektoren $\vec{E}$ durch Auswerten von (7) ermittelt werden. Die Rechnung selbst geschieht durch Verwendung der drei Knotenpunktswerte des Potentials $U$ eines Dreiecks fuir dasselbe, so wie durch die hellgrauen Pfeile in der Abb. 10 angedeutet und in Abb. 11 anschaulich dargestellt. In den nächsten beiden Schritten muss man nun

1. von den in den Schwerpunkten gegebenen Daten reinterpolieren auf die Knotenpunktsdaten (graue Pfeile in der Abb. 10 - wofür oben ein mögliches Verfahren [Gl.(15)] vorgestellt wurde) und dann

2. diese Daten wiederum partiell differenzieren, um auf die Divergenzen der elektrischen Erregung zu kommen (die mit den dunklen Pfeilen angedeuteten Wege - die Farbgebung: Hellgrau, grau und dunkelgrau soll dabei die wachsende Unsicherheit in dem Interpolationsprozess andeuten).

Es sind also Richtungsableitungen 2. Ordnung in $U$ aus den Lösungen der Gl. (10) für (2) numerisch zu ermitteln. Um dies zu können, müssen die ersten Ableitungen, d. i. hier die Berechnung von nach (7), möglichst präzise erfolgen. Das Problem weiter zu erklären, soll mit der Abb. (11) versucht werden:

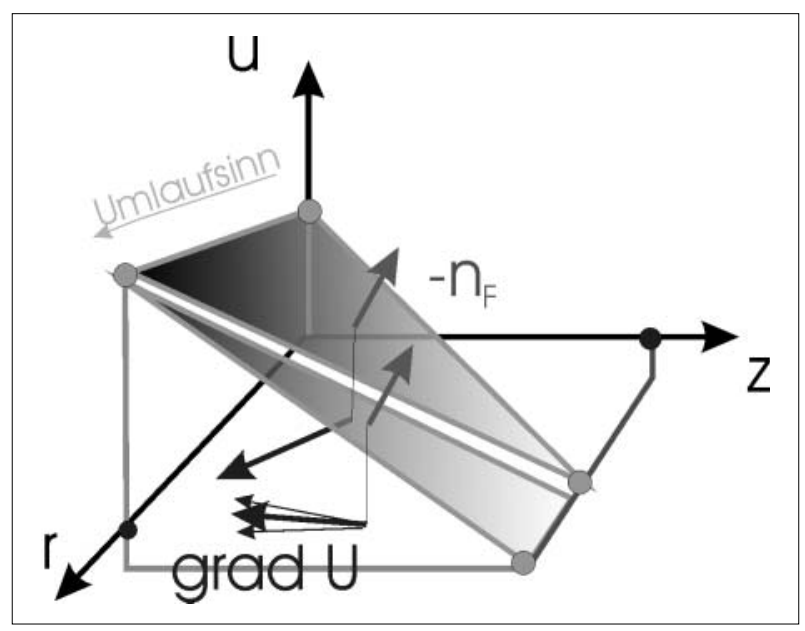

Abb. 11: Zur Bildung des Gradienten in einem Triangulationsnetz

Man kann sich die Triangulation als über die $r$-z-Ebene gelegte Fläche mit den in den Knotenpunkten durch den Wert des Potentials gegebenen Höhen denken. Auf jeder dieser Flächen kann der dazu normale Vektor errichtet werden. Die Projektion dieses Vektors in die $r$-z-Ebene ist dann aber der Gradient - der Vektor, der in die Richtung des steilsten Anstieges weist.

Bei dem in Abb. (11) gezeigten oberen Dreieck wird der Vektor des Gradienten so gebildet, dass er sicher in die Richtung des steilsten Anstieges zeigt. Bei dem unteren Dreieck ist leicht einzusehen, dass bezuiglich der $r$-Komponente der Fehler immer größer wird, je spitzer dieses Dreieck ist. Will man solche Missweisungen vermeiden, dann muss man an die Triangulation (auch noch aus anderen Gründen) die Forderung der möglichsten Gleichseitigkeit aller Dreiecke stellen. Natürlich wird diese sich nicht vollständig herstellen lassen, es stellt sich aber die Frage: Wie gut lässt sie sich (mit vertretbarem Aufwand) herstellen?

\subsection{Innerhalb der erschwinglichen Software}

Innerhalb des Softwarepaketes MATLAB gibt es eine Routine zur Verbesserung der Qualität eines generierten Netzes. Der geringe Nutzen derselben dürfte sein, das sie lediglich an den inneren Punkten (s. u.) manipuliert, aber alle Randpunkte (bzw. Punkte auf Grenzflächen) als unveränderlich betrachtet. Man erhält in der Regel Netze, deren Qualität nach Aussagen der in [3] zitierten mathematischen Literatur gut ist. Wir haben aber festgestellt, dass dies nicht ausreichend ist, um eine zweite Ableitung des Potentials zu bilden.

\subsection{Mit Optimierungsverfahren}

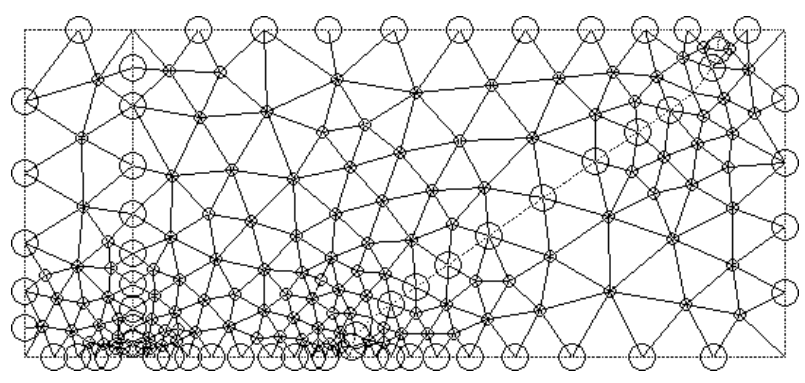

Abb. 12: Optimierungsvariablen des Ausgangsnetzes mit einigen $q_{i}<0.7$ 
Die Abb. 12 zeigt die Freiheit und die Grenzen, die gegeben sind, hochqualitative Netze aus einer vorgegebenen Triangulation ${ }^{\mathrm{IV}}$ zu erzeugen: Alle mit einem kleinen Marker versehenen Punkte sind die $n_{I}$ inneren Punkte. Jeder dieser Punkte kann (im Rahmen vernünftiger Grenzen natürlich) eigentlich beliebig in $z$ - und $r$-Richtung verschoben werden. Auf den Rändern gibt es $n_{R}$ Punkte (mit großen Marken versehen), die entlang dieser Ränder (natürlich auch nur innerhalb gewisser Grenzen) verschoben werden können. Jede Änderung dieser $2 * n_{I}+n_{R}$ Optimierungsvariablen $x$ bewirkt eine Änderung der $q_{i}$ Qualitätswerte der $n_{T}$ Dreiecke der Triangulation. Zur Bewerkstelligung der Aufgabe benötigt man noch eine Zielfunktionen (in Anlehnung an [3]):

Darin ist $A_{i}$ die Fläche eines Triangulationsdreiecks. $l_{i 1 . .3}$ sind die Seitenlängen desselben. Der Subtrahend ist ein Maß aus [0,1] für die Qualität eines Dreiecks. Für gleichseitige Dreiecke ist es 1 . Die beste Triangulation wäre also die, fuir die $F_{i}=0_{\forall i}$ gilt. Eine solche Forderung ist aber (bei beliebigen Geometrien) nicht zu erfüllen. Optimierungsziel kann aber sein,

$$
\min _{x} \max _{\left\{F_{i}\right\}}\left\{F_{i}(x)\right\}
$$

In Worten: Finde einen solchen Satz von Optimierungsvariablen $x$, dass das Maximum der Funktionswerte $F_{i}$ minimal wird. Dies ist ein sogenanntes minimax-Problem. Kann mantes $3 \ddot{A}$ sten, dann ist evident, dass alle

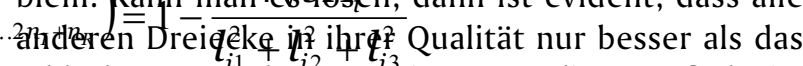
schlechteste sein können. Ḋie Lösung dieser Aufgabe ist Standard bei den angebotenen Optimierungsalgorithmen. Das Ergebnis der Anwendung auf das hier behandelte Beispiel zeigt die Abb. 13.

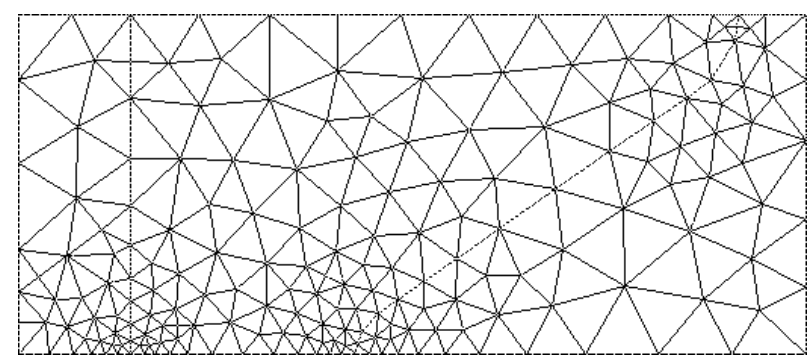

Abb. 13: Nach minimax optimiertes Ausgangsnetz, alle $q_{i}<0.84$

Hatte das Ausgangsnetz Dreiecke mit Qualitäten < 0.7, dann hat das schlechteste jetzt eine Qualität von etwa 0.84 (vergleicht man beide Abbildungen, so erkennt man durchaus Verschiebungen). Es ist leicht einsichtig, dass sich mit der Verfeinerung des Netzes (= Erhöhung der Variablenzahl) die Qualität des Netzes auch weiter erhöhen lässt. Der Rechenaufwand wächst aber mit der Zahl der Variablen exponentiell, so dass dem minimax-Verfahren hier schnell Grenzen gesetzt sind.

Als brauchbar an einem solchen (recht groben, aber hochoptimierten) Ausgangsnetz erweist sich aber, dass man bei Verfeinerung des (gesamten) Netzes nun kaum eine Verschlechterung der Qualität mehr erhält, da alle Dreiecke nahezu gleichseitig sind. Nur ist es nicht finales
Ziel, das gesamte Netz zu verfeinern, sondern nur Teile dessen (hier insbesondere die Umgebung der Spitze). Um nun auch noch größere Netze in vertretbaren Zeiten in gute Qualität zu bringen, muss man das Ziel etwas unschärfer fassen - oder die Forderung mildern zu:

$$
\min _{x} \sum_{i=1}^{n_{T}} F_{i}(x)^{2 m}
$$

Für $m \rightarrow \infty$ entspricht dies (mathematisch) der minimaxForderung. Für $m=1$ einer Minimierung des quadratischen Mittels der Zielfunktionen.

Wie alle analytisch begründeten Verfahren der nichtlinearen Optimierung riskiert man bei deren Anwendung in Abhängigkeit von den gewählten Startwerten, auf einem lokalen Extremum zu landen. Um so höher man in (28) $m$ wählt, mit um so größerer Sicherheit landet man im Ergebnis eines solchen Optimierungsversuches auch auf einem solchen.

\subsection{Mit Evolutionsverfahren}

Eine der frühesten Übersichtsarbeiten zu diesem einfachen Prinzip ist [4]. Ursache der Entwicklung solcher Verfahren war die Suche nach optimalen Lösungen von Permutationsproblemen (Rundreiseproblem mit N! Varianten $N$ Orte nacheinander aufzusuchen - also von extrem großen Problemen). Grundlegende Idee war, dies nach dem Prinzip der Selektion und Mutation der Biologie zu tun. Die algorithmische Implementation dieser Idee ist so einfach, dass hier kurz skizziert werden kann: 1. Wähle einen Startvektor der Optimierungsvariablen.

2. Ändere diesen Startvektor zufällig mit einem normalverteilten Wert zu einer vorher als Parameter gegebenen Standardabweichung s.

3. Werte die Zielfunktion (hier Gl. (27) mit (26)) aus.

4. Nun teste: Ist man dem Ziel näher gekommen, dann nimm den erhaltenen Vektor als neuen Startvektor, und setze damit bei 2 . fort. So nicht, dann sind (so die Annahme) die Mutationen zu heftig, um einem Trend zum Durchbruch zu verhelfen: Verringere die Streuung der Mutationen (senke die Temperaturv) und wiederhole mit dem alten Startvektor ab 2 .

Merkwürdigerweise, so das Absenken der Temperatur nicht zu schnell erfolgt, tendiert dieser Algorithmus dahin, das globale Minimum einer Aufgabe mit vielen Variablen zu finden. Hier wurde dieser freezen-Algorithmus bei der Optimierung von Triangulationen zwar ein wenig modifiziert, im Wesentlichen arbeitet er aber so. Als empirisch nuitzliches Vorgehen (bei hochdimensionalen Traingulationsnetzen) hat sich nun herausgestellt folgendermaßen vorzugehen:

1. Suche eine nach (28) gute Lösung des Minimierungsproblems mit moderatem Parameter $m$.

2. Da diese Lösung i. A. auf ein lokales Minimum fuihrt, fuihre einige (1000) Iterationsschritte nach der Evolutionsstrategie aus.

3. Wiederhole ggf. 1 . und 2.

Wir haben so durchweg Netze erzeugen können, deren Qualitätsparameter grundsätzlich $>0.8$ sind; wir können also einer Diskussion der Qualität der verwendeten Netze prinzipiell aus dem Weg gehen. 


\section{Die Berechnung konsistenter Flüsse - oder beste Interpolation}

Für die folgenden Untersuchungen ist der oben beschriebene Korrekturmechanismus ausgeschaltet. Es interessieren nur die nach Gl. (13) erhaltenen numerischen Lösungen von (10), und zwar ausschließlich unter dem Gesichtspunkt der Berechnung der Werte von (5) bzw. (24) im Entladungsspalt. Dazu ist in der rechten Seite von (10) für einen Teil des Spaltes eine rechteckförmige Raumladungsdichte von $10^{7} \mathrm{~cm}^{-3}$ eingesetzt. Nach (14) sind dann die Komponenten der elektrischen Feldstärken $E_{r}$ und $E_{z}$ berechnet, diese dann mit (16) auf die Knotenpunkte interpoliert und so $E_{r}{ }^{K}$ und $E_{z}{ }^{K}$ ermittelt worden. Völlig analog zur Gl. (14) - der Bildung des Gradienten - berechnet man nun die Divergenz (in Zylinderkoordinaten) nach

$$
\operatorname{div} \vec{E}=\frac{1}{r_{s}} \boldsymbol{G}_{r}\left(r_{K} E_{r}^{K}\right)+\boldsymbol{G}_{z} E_{z}^{K} .
$$

Darin ist $r_{s}$ die radiale Koordinate des Schwerpunktes des Dreiecks, für welches die Quellstärke berechnet werden soll, und $r_{K}$ die des Knotenpunktes, an dem $E_{r}{ }^{K}$ gegeben ist. Multipliziert man dieses Ergebnis noch mit $\varepsilon_{0}$, dann muss man wieder $\rho$, die vorgegebene Raumladungsdichte, erhalten. Man erhält aber $\rho_{\text {bak }}$, so wie in Abb. 14 gezeigt.

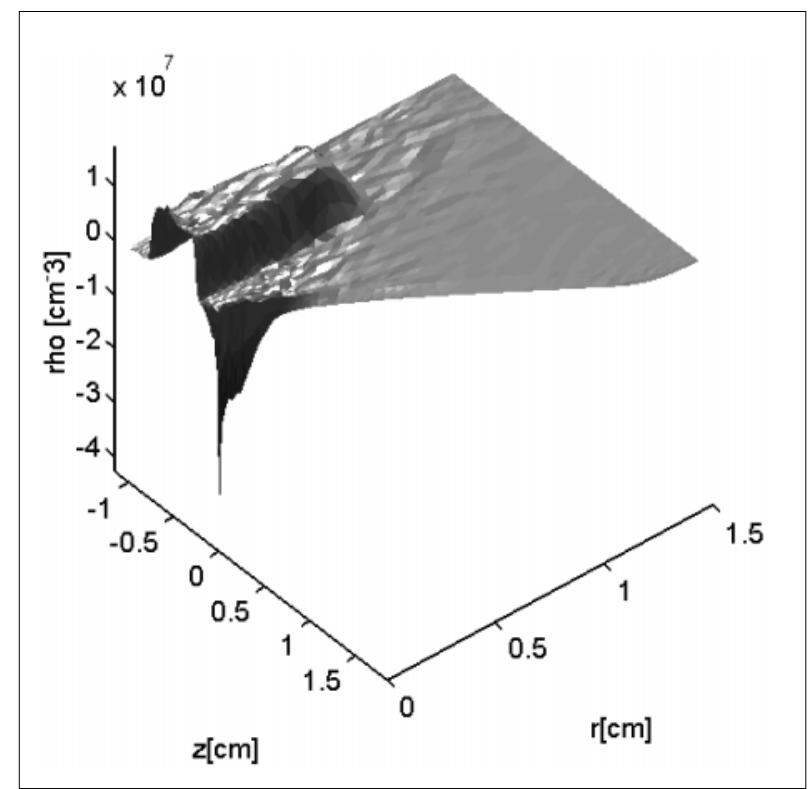

Abb. 14: Aus vorgegebener Raumladung zurückgerechnete Quelldichte $\rho_{\text {bak }}$

Was man erhält, ist stellenweise nun kein Fehler mehr, sondern einfach nur ein unsinniges Ergebnis (insbesondere in der Nähe der Spitze und der Symmetrieachse). Ursache ist die nach Gefühl konstruierte Rückinterpolation von aus den Knotenpunkten erhaltenen Daten für ein Triangulationselement auf die Knotenpunkte selbst wieder. Wenn nun aber die Auswahl einer Interpolationsformel weitgehend eine philosophische Frage zu sein scheint, dann kann man das Philosophieren über eine solche (z. B. Gl. (15)) vermeiden, wenn man ganz einfach nach der besten Interpolationsformel fragt.
In der Abb. 10 hatten wir mit den dunkelgrauen Pfeilen angedeutet, wie man mit der (philosophisch gewählten) Interpolationsformel (15) aus den einen Punkt umgebenden Dreiecken einen Knotenpunktwert ermittelt. Eine gute Interpolation wäre nun eine solche, bei der die (linearen Rück-)Rückinterpolation aus den Knotenpunktwerten auf den Schwerpunkt des Dreiecks wieder den dort gegebenen Wert (aus Gl.(14)) und hier mit bezeichnet) gibt. Wir hätten also für eine gute Interpolation aus den Schwerpunkten auf die Knoten an die Knotenpunktwerte $E_{1 \ldots 3}$ die Forderung zu stellen:

1.

Diese Forderung ist für jedes der $n_{T}$ Dreiecke in der Triangulation zu stellen. Da es aber im Allgemeinen in einer Triangulation mehr Dreiecke als Punkte $n_{K}$ gibt (jeder Punkt ist in der Regel Eckpunkt von mehr als drei Dreiecken $^{\mathrm{VI}}$ - man betrachte dazu z. B. Abb. 12), ist das System (30) nicht exakt zu befriedigen. Nun sind dies auch wenn in der Regel schon zu viele (sich also (30) nicht exakt befriedigen lässt) - nicht die einzigen Forderungen, die wir an eine gute Interpolation zu stellen hätten. In jeder Triangulation gibt es $n_{E}$ Seiten, die jeweils zwei Punkte miteinander verbinden. Auf jeder dieser Seiten lassen sich aus den zu seinen an den Endpunkten gegebenen (besser noch nicht gegebenen) Vektoren $E_{1}$ und $E_{2}$ die zu dieser Seite zugehörigen Normalkomponenten an den Endpunkten derselben berechnen (in der Abb. 15 exemplarisch für eine Seite mit $E_{n 1}$ und $E_{n 2}$ bezeichnet).

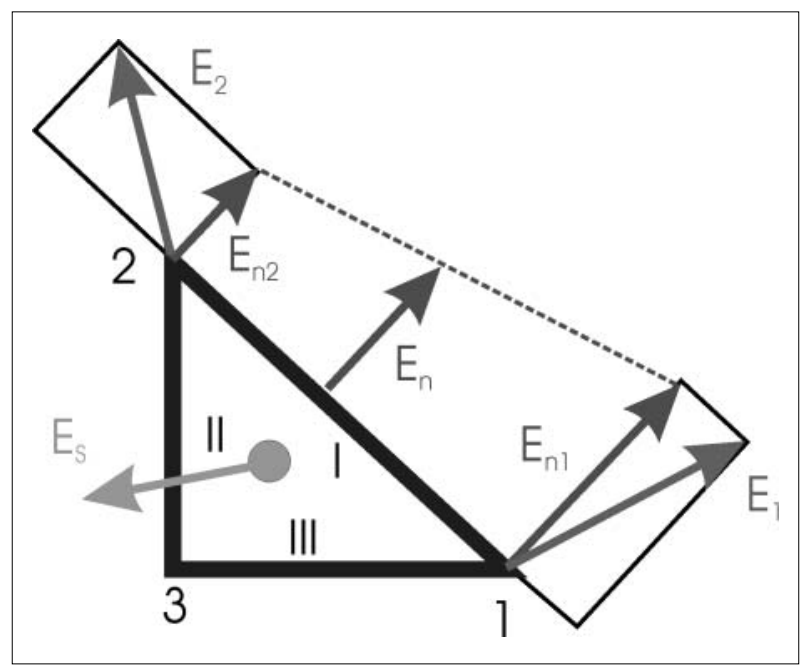

Abb. 15: Einige Bezeichnungen

Unterstellt man längs der Seite eines solchen Dreiecks Interpolierbarkeit, dann erhält man als mittleren elektrischen Fluss durch diese Seite

$$
D_{n}=\varepsilon_{r}\left(w_{1} E_{n 1}+w_{2} E_{n 2}\right)=\varepsilon_{r} E_{n} .
$$

$w_{1}$ und $w_{2}$ sind Integrationsgewichte, die hier bei linearer Interpolation fuir das Problem in Zylinderkoordinaten gegeben sind als: 


$$
\begin{aligned}
& w_{1}=\pi l_{12} \frac{2 r_{1}+r_{2}}{3} \\
& w_{2}=\pi l_{12} \frac{r_{1}+2 r_{2}}{3}
\end{aligned} .
$$

$r_{1}$ und $r_{2}$ sind die radialen Koordinaten der Punkte 1 und $2, l_{12}$ die Länge der Seite (Details siehe in [2]). Dabei ist z. B. $E_{n 1}=\vec{E}_{1} \cdot \vec{n}_{I}$ mit dem Wert aus der Forderung 1 aus (30) zu nehmen. Die Verknüpfung bezüglich der in (30) fuir jedes Dreieck der Triangulation aufgestellten Bedingungen ergibt sich nun aus der Forderung:

$$
\text { 2. } \frac{A_{I} D_{n I}+A_{I I} D_{n I I}+A_{I I I} D_{n I I}}{V_{T}}=\rho \text {. }
$$

Das ist, dass die Summe der elektrischen Flïsse durch jede der Seiten $A_{I \ldots I I I}$ eines Teilvolumens $V_{T}$ der Diskretisierung, die Quellstärke desselben ergeben muss, was in dieser Schreibweise bei $\lim _{V_{T} \rightarrow 0}$ lediglich Gl. (8) in integraler Formulierung ist. $\mathrm{Zu}$ diesen fundamentalen Formulierungen innerhalb des Volumens kommen noch einige marginale aus den Rand- und Grenzflächenbedingungen. In der Summe ergeben all diese Forderungen ein wenn auch sehr großes, so doch nur schwach besetztes, stark überbestimmtes lineares System, welches man im Sinne der Minimierung der Summe der Fehlerquadrate löst. So findet man an Stelle der gefühlsmäßig aufgestellten Interpolationsformel (15) die beste - in dem Sinne, dass sie die Fehler bzgl. der aufgestellten Forderungen minimiert. Damit haben wir das in der Abb. 16 gezeigte Ergebnis der oben erläuterten Rückrechnung erreicht.

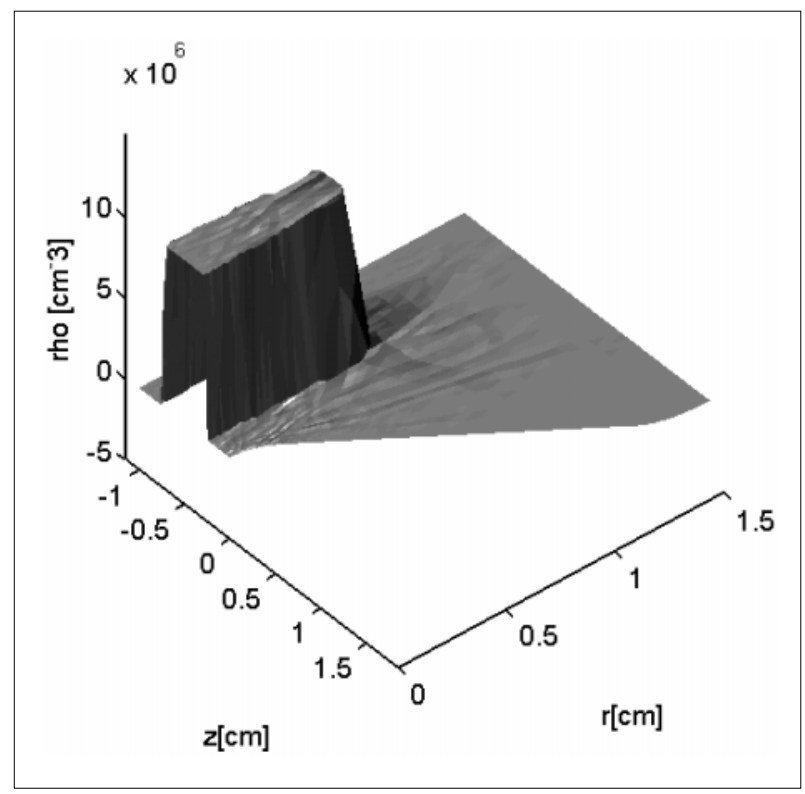

Abb. 16: Aus vorgegebener Raumladung zurückgerechnete Quelldichte mit der besten Interpolation

Um die Akzeptierbarkeit der so gewonnen Rückrechnung zu zeigen, ist das hier zur Illustration genutzte Beispiel in einem wesentlich gröberen Netz gerechnet worden, sonst wären die Unterschiede zwischen den in der Abb. 17 gezeichneten besten und den nach (14) berechneten Feldstärkevektoren nicht in einer solchen Darstellung zu erkennen. ${ }^{\text {II }}$

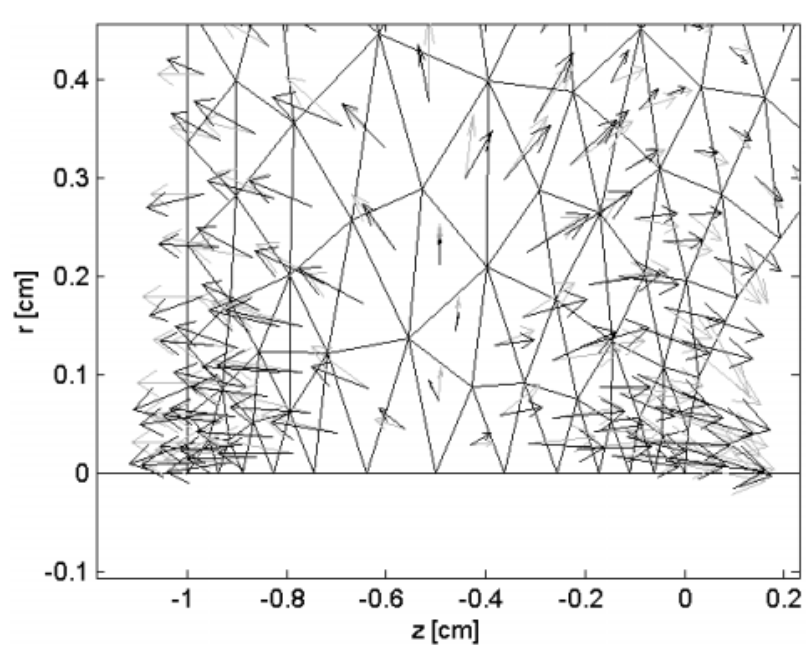

Abb. 17: Zur Interpretation der Korrekturen. Die hellgrauen Vektoren sind die nach (14) berechneten und in den Schwerpunkt eines jeden Dreiecks gesetzten. Die schwarzen Vektoren sind die sich aus der besten Interpolation dafür ergeben Habenden.

Mit denen als Nebenprodukt erhaltenen Werten von $E_{n 1}$ und $E_{n 2}$ lassen sich nun die Normalkomponenten der Driftgeschwindigkeit an den Endpunkten jeder Seite der Triangulation berechnen. Den mittleren Fluss bildet man dann analog zu (31) und kann nun endlich den Ausdruck (5) numerisch konsistent berechnen und beginnen Gl. (1) zu lösen.

\section{Danksagung}

Die hier dargestellten Ergebnisse sind erzielt worden durch Förderung des BMBF/VDI (FKZ: 13N 7549 / 4) innerhalb eines Verbundprojektes zwischen den Partnern:

- Institut für Umwelttechnologien $\mathrm{GmbH}$, Berlin

- Process Technology Development GmbH, Berlin

- Rafflenbeul Ingenieure GmbH, Frankfurt/M.

- Fachhochschule Merseburg

- Technische Fachhochschule Wildau

\section{Quellennachweis}

[1] Jahresbericht 2001 des Teilvorhabens Experimentell gestiitzte Modellierung von Gasentladungen im Verbundvorhaben : Grundlagen der nichtthermischen Plasmachemie zur Abluftreinigung, FKZ: 13N 7549 / 4

[2] Jahresbericht 2002 ebenda.

[3] Das von uns verwendete Softwarepaket MATLAB verzeichnet verschiedene Quellen - in den Handbüchern sowie in den Quellcodes - bzgl. mathematischer Verfahren und der Implementation derselben. Wir verweisen in diesem Beitrag - so wir darauf Bezug nehmen - lediglich auf diese Quelle, ohne sie zu Untersetzen.

[4] Rechenberg, Optimierung technischer Systeme nach Prinzipien der biologischen Evolution. PhD thesis, TU Berlin, Fakultäat für Maschinenwesen, Oktober 1973. 


\section{Anmerkungen}

I Bei Systemen, deren eine Elektrode metallisch ist oder die nicht solch hohe Kapazität aufweisen, wie das hier betrachtete, kämen noch die Gleichungen des äußeren elektrischen Kreises hinzu.

II Eine weitere wesentliche aus (8) folgende Bedingung ist: Es muss uiberall die Stetigkeit der Tangentialkomponenten der elektrischen Feldstärke gewährleistet sein. Dies wird von der FEM erfuillt. Deshalb ist dies hier nirgends weiter angesprochen.

III Hier sowie an allen anderen Orten dieser Arbeit sind die Ladungen in Einheiten der Elementarladung angegeben. Flächenladungen also in $\mathrm{cm}^{-2}$ und Raumladungen in $\mathrm{cm}^{-3}$.

IV Wir haben keine Vergleiche von verschiedenen Triangulationsmethoden anstellen können und nur die in [3] gegebenen Möglichkeiten genutzt.

V In der statistischen Thermodynamik entspricht die Temperatur in der Maxwell-Boltzmann-Verteilung dem Quadrat der Standardabweichung in der Normalverteilung. Deshalb für diesen Algorithmus auch die Bezeichnung freezen algorithmen.

VI Das dies nicht anders geht, ist einleuchtend. Man triangularisiere ein Dreieck mit wenigstens einem inneren Knotenpunkt. Dieser ist dann immer gemeinsamer Punkt von mindestens drei Dreiecken.

VII Die nach (14) berechnete Feldstärke gilt jeweils für das ganze Dreieck, aus dessen Eckwerten sie ermittelt wurde. Wir argumentierten, dass dieser Wert im Schwerpunkt am wahrscheinlichsten ist. Der jetzt ermittelte Wert ist ein solcher, der alle Forderungen des Systems möglichst bestens zu befriedigen sucht.

\section{Autoren}

Dipl. Phys. Harald J. Beyer

E-Mail: hjbeyer@igw.tfh-wildau.de

Dr.-Ing. Andreas Harendt

E-Mail: andreas@harendt.de

Prof. Dr. rer. nat. Siegfried Rolle

E-Mail: rolle@pt.tfh-wildau.de

Technische Fachhochschule Wildau

Fachbereich Ingenieurwesen/Wirtschaftsingenieurwesen Institut für Plasma- und Lasertechnik

Tel. (0 33 75) 508-126

Internet: www.iplt.de 